\title{
STUDY OF A PREY-PREDATOR DYNAMICS UNDER THE SIMULTANEOUS EFFECT OF TOXICANT AND DISEASE
}

\author{
SUDIPA SINHA ${ }^{1}$, O.P.MISRA ${ }^{2}$ AND JOYDIP DHAR ${ }^{3 *}$
}

\begin{abstract}
A mathematical model is proposed to study the simultaneous effects of toxicant and infectious disease on Lotka-Volterra prey-predator system. It is considered in the model that only the prey population is being affected by disease and toxicant both, and the susceptible and infected prey populations are being predated by predator. All the feasible equilibrium of the model are obtained and the condition for the existence of interior equilibrium point is also been determined. The criteria for both local stability and instability involving ecotoxicological and epidemiological parameters are derived. The global stability of the interior equilibrium point is discussed using Lyapunov's direct method. The results are compared with the case when environmental toxicant is absent. Moreover, threshold conditions depending upon toxicant, disease and predation related parameters for the non-linear stability of the model is determined. Finally, the numerical verifications of analytic results are carried out.
\end{abstract}

\section{INTRODUCTION AND PRELIMINARIES}

The classical Lotka-Volterra model is well known among the ecologists. Also the study of the epidemic models accounting for the spread of disease in a population and the models to study the effect of pollutants emitted into environment on existence of population has been done by previous researchers. Our purpose is to study the combined system. There has been growing interest in the study of disease in a prey-predator system. The invasion of a resident prey-predator or host-parasite system by a new strain of parasite has been studied by [2]. The conditions for long term persistence of two populations growing exponentially [8], and each affected by disease has been studied by [16]. [5] studied the role of more

Date: Received:17 July 2008.

${ }^{*}$ Corresponding author.

2000 Mathematics Subject Classification. 92, 92A.

Key words and phrases. Prey-Predator Dynamics, Disease, Toxicant, Stability, Simulation. 
complicated models, including multispecies interactions, where an ecological view point is taken that the host density itself becomes a dynamical variable. The coexistence and stability regulated by inter and intra-specific infection has been investigated analytically by [6], but, the numerical investigations do not show existence of stable limit cycle. Some more models discussing infection in populations are studied in [4, 6, 3], and disease transmission problems and epidemiological models are studied in [7, 13, 17, 18, 19, 20, 1, 23. In present days, the effect of pollutant on the survival or persistence of the population is also a major problem. The industrial units and many man-made projects emit pollutant in the form of Sulphur Dioxide, Nitrogen Dioxide, Carbon Monoxide, Hydrocarbons, Fluorine, Fly ash etc. The pollutants effect the ecosystem in general and plant in particular [14, 24, 11]. In recent years, the effect of toxicants on biological species has also been investigated [25, 26, 12, 27, 22, 21, 11, 10]. The effect of toxicants shown in various models is that, it decreases the growth rate of biological species as well as its carrying capacity. A single species model in polluted closed environment with toxicant input at fixed moment has been studied [21] and it has been shown that the population will extinct if the impulsive period is less than some critical value otherwise population will persist. Thus, to study the simultaneous effect of pollution stress and the effect of infection in an interacting species is of a great importance to derive the feasible situations of an ecosystem.

Keeping in view the ecological relevant questions of species-survival and the long term behavior of the system, in the present study, we have proposed a mathematical model which has been constructed by combining three basic models dealing with prey-predator interaction, disease spread (SI model) and the effect of environmental pollution on single prey species. In the SI model, it is considered that prey-population is affected by infectious disease. This paper is organized as follows : In section 2 we have given a sub-model, i.e., Model 1 and the main model,i.e., Model 2. In section 3 we have done the boundedness and the dynamical analysis of Model 1 and Model 2 respectively. In section 4, numerical results and graphical illustration has been given. Finally, in the last section a brief conclusion of the proposed model has also been included.

\section{Mathematical Model}

Here, we have considered a general prey-predator system, in which prey is subjected to some disease. In this way, the prey population is subdivided into two parts one of which is susceptible prey population and another one is infected prey population; represented by $x_{1}(t)$ and $x_{2}(t)$ state variables respectively. Predator population $y(t)$ is assumed to feed on both the susceptible and infected prey population with different predation rates. Thus, to study the effect of spread of disease on the prey population, which is also assumed to be predated by some predator species, we have proposed the following model:

\section{Model 1:}

$$
\frac{d x_{1}}{d t}=\theta-\beta x_{1} x_{2}-\alpha_{1} x_{1} y-d_{1} x_{1}
$$




$$
\begin{gathered}
\frac{d x_{2}}{d t}=\beta x_{1} x_{2}-\alpha_{2} x_{2} y-d x_{2} \\
\frac{d y}{d t}=\alpha_{1} x_{1} y+\alpha_{2} x_{2} y-d_{3} y
\end{gathered}
$$

The above ordinary differential equations are associated with the following initial conditions: $x_{1}(0)=x_{10}>0, x_{2}(0)=x_{20}>0, y(0)=y_{0}>0$; where, $\theta$ is recruitment rate of susceptible prey, $\alpha_{1}, \alpha_{2}$ are the predation rates among the susceptible and infected preys, $\beta$ is the disease contact rate, $d_{1}, d_{3}$ are the natural death rates in susceptible prey and predator populations, and $d=\left(d_{2}+h\right)$ is the net death rate in infective prey population which is sum of natural and disease induced death rates.

Now, we consider that only the prey population is being affected by toxicant in the system described by equations $(2.1)-(2.3)$. Let $U(t)$ be the toxicant concentration in the population $x(t)=\left(x_{1}(t)+x_{2}(t)\right)$. $C(t)$ is the environmental concentration of toxicant. After incorporating the effect of toxicant in the model 1, we get the following model:

\section{Model 2:}

$$
\begin{gathered}
\frac{d x_{1}}{d t}=\theta-\beta x_{1} x_{2}-\alpha_{1} x_{1} y-r_{1} U x_{1}-d_{1} x_{1} \\
\frac{d x_{2}}{d t}=\beta x_{1} x_{2}-\alpha_{2} x_{2} y-r_{2} U x_{2}-d x_{2} \\
\frac{d y}{d t}=\alpha_{1} x_{1} y+\alpha_{2} x_{2} y-d_{3} y \\
\frac{d C}{d t}=Q-\alpha C-\delta C\left(x_{1}+x_{2}\right) \\
\frac{d U}{d t}=\delta C\left(x_{1}+x_{2}\right)-m U
\end{gathered}
$$

The above ordinary differential equations are associated with the following initial conditions: $x_{1}(0)=x_{10}>0, x_{2}(0)=x_{20}>0, y(0)=y_{0}>0, U(0)=U_{0}>$ $0, C(0)=C_{0}>0$.

Along with the parameters of model 1, we have following additional parameters in model 2:

$m$ is the natural wash out rate of the toxicant from the organism, $r_{1}, r_{2}$ are the rates at which susceptible and infected prey are decreasing due to toxicant, $\delta$ is uptake rate of toxicant by organism, $\alpha$ is the natural depletion rate of the environmental toxicant, and $Q$ is the exogenous input rate of the toxicant in the environment. Basically, by means of above model we want to study the effect of environmental pollution on a prey-predator system when prey population is already subjected to some disease.

In the next section we have studied the boundedness and the dynamical behaviour of the both the models. 


\section{Boundedness And Dynamical Behaviour}

3.1. Analysis of Model 1: In this section, it has been established that, all the solutions of Model 1 are bounded in a positive orthant in $R_{+}^{3}$. The boundedness of solutions of Model 1 is given by the following lemma.

Lemma 3.1. All the solutions of Model 1, will lie in the region $B_{1}=\left\{\left(x_{1}, x_{2}, y\right) \in\right.$ $\left.R_{+}^{3}: 0 \leq x_{1}+x_{2}+y \leq \frac{\theta}{\theta_{1}}\right\}$ as $t \longrightarrow \infty$, for all positive initial values $\left(x_{10}, x_{20}, y_{0}\right) \in$ $R_{+}^{3}$; where $\theta_{1}=\min \left(d_{1}, d, d_{3}\right)$.

Proof. : Let us consider the following function:

$$
W(t)=x_{1}(t)+x_{2}(t)+y(t)
$$

from equations (2.1)-(2.3), and if $\theta_{1}=\min \left(d_{1}, d, d_{3}\right)$ then we get:

$$
\dot{W}(t) \leq \theta-\theta_{1} W
$$

then by usual comparison theorem [Hale, 1980], we get the following expression as $t \longrightarrow \infty$ :

$$
W(t) \leq \frac{\theta}{\theta_{1}} \text {, and hence, } x_{1}(t)+x_{2}(t)+y(t) \leq \frac{\theta}{\theta_{1}}
$$

This completes the proof of lemma.

Now, we will discuss the stability of the boundary equilibrium points and interior equilibrium point. Model 1 has the following set of feasible set of boundary equilibrium points: $E_{0}=(0,0,0), E_{1}=\left(\theta / d_{1}, 0,0\right), E_{2}=\left(d / \beta, \frac{\beta \theta-d_{1} d}{d}, 0\right), E_{3}=$ $\left(d_{3} / \alpha_{1}, 0, \frac{\theta \alpha_{1}-d_{1} d_{3}}{d_{3}}\right)$ and non-trivial equilibrium point:

$E_{4}=\left(\frac{d_{3}-\alpha_{2} x_{2}^{*}}{\alpha_{1}}, \frac{d_{3}}{\alpha_{2}}+\frac{\theta \alpha_{1}}{d \alpha_{1}-d_{1} \alpha_{2}-\beta d_{3}}, \frac{\beta d_{3}-d \alpha_{1}-\beta \alpha_{2} x_{2}^{*}}{\alpha_{1} \alpha_{2}}\right)$. Now, we will discuss the dynamical behavior of the Model 1 .

- $E_{0}$ is the trivial equilibrium point. Corresponding to this equilibrium point we have the eigen values $-d_{1},-d,-d_{3}$. Thus, this point is attracting.

- Corresponding to the equilibrium point $E_{1}$ we have the eigen values $-d_{1},(\beta \theta-$ $\left.d d_{1}\right) / d_{1},\left(\alpha_{1} \theta-d_{1} d_{3}\right) / d_{1}$. This point is also stable in $x_{1}$ direction and may be stable or unstable in $x_{2}-y$ plane depending up on system parameters.

- The equilibrium point $E_{2}$ is feasible when $\theta>\frac{d_{1} d}{\beta}$, biologically, which means that the recruitment rate in the susceptible population should be greater than some positive threshold value, which depends upon disease contact rate. The eigen values corresponding to this equilibrium point in $x_{1}$ and $x_{2}$ directions are real negative roots of the following characteristic polynomial:

$$
\lambda^{2}+\left(\beta \frac{\beta \theta-d_{1} d}{d}+d_{1}\right) \lambda+\beta\left(\beta \theta-d_{1} d\right)=0
$$

and eigen value in $y$ direction is $\alpha_{1} \beta / d+\alpha_{2} \frac{\beta \theta-d_{1} d}{d}-d_{3}$. This point is attracting in $x_{1}-x_{2}$ plane if $\beta \theta>d d_{1}$.

- The equilibrium point $E_{3}$ is feasible when $\theta>\frac{d_{1} d_{3}}{\alpha_{1}}$ and corresponding to this point, we get the following characteristic equation:

$$
\left(\lambda^{2}+\left(\alpha_{1} \tilde{y}+d_{1}\right) \lambda+\alpha_{1}^{2} \tilde{x}_{1} \tilde{y}\right)\left(\lambda-\left(\beta \tilde{x_{1}}-\alpha_{2} \tilde{y}-d\right)\right)=0
$$


$E_{3}$ is attracting in $x_{1}-y$ plane and may be stable or repulsive in $x_{2}$ direction depending up on system parameters.

- Finally, the equilibrium point $E_{4}$ is feasible when $d_{3}>\alpha_{2} x_{2}^{*}, \beta d_{3}>d \alpha_{1}+$ $\beta \alpha_{2} x_{2}^{*}$, and $\left(\beta+d_{1}+a \alpha_{1}\right) d_{3}>\theta \alpha_{3}$ and corresponding to this point, we get the following characteristic equation:

$$
\lambda^{3}+a_{1} \lambda^{2}+a_{2} \lambda+a_{3}=0
$$

where $a_{1}=\left(\beta x_{2}^{*}+\alpha_{1} y^{*}+d_{1}\right), a_{2}=\left(\beta^{2} x_{1}^{*} x_{2}^{*}+\alpha_{1}^{2} x_{1}^{*} y^{*}+\alpha_{2}^{2} x_{2}^{*} y^{*}\right)$ and $a_{3}=\alpha_{2}^{2} x_{2}^{*} y^{*}\left(\beta x_{2}^{*}+\alpha_{1} y^{*}+d_{1}\right)$. Then, by Routh-Hurwitz criteria $E_{4}$ is locally asymptotically stable if $a_{1}>0, a_{2}>0, a_{3}>0$ and $a_{1} a_{3}-a_{2}>0$.

Now, we will discuss the global stability of interior equilibrium point $E_{4}$. The global stability has been achieved using Lyapunov's direct method. The global stability of interior equilibrium point $E_{4}$ is determined by the following theorem.

Theorem 3.2. The interior equilibria $E_{4}$ of the system described in Model 1, is globally asymptotically stable for all positive initial values $\left(x_{10}, x_{20}, y_{0}\right) \in R_{+}^{3}$.

Proof. Let we consider the following positive definite function :

$$
V_{1}\left(x_{1}, x_{2}, y\right)=\sum_{i=1}^{2} w_{i}\left(x_{i}-x_{i}^{*}-x_{i}^{*} \ln \frac{x_{i}}{x_{i}^{*}}\right)+w_{3}\left(y-y^{*}-y^{*} \ln \frac{y}{y^{*}}\right)
$$

then the time derivative of above function is given as:

$$
\dot{V}_{1}(t)=w_{1} z_{1} \frac{\dot{x_{1}}}{x_{1}}+w_{2} z_{2} \frac{\dot{x_{2}}}{x_{2}}+w_{3} z_{3} \frac{\dot{y}}{y}
$$

where, $z_{1}=\left(x_{1}-x_{1}^{*}\right), z_{2}=\left(x_{2}-x_{2}^{*}\right)$, and $z_{3}=\left(y-y^{*}\right)$.

Now from equations (2.1)-(2.3) and from equation (3.2), we get:

$\dot{V}_{1}(t)=-\frac{\theta w_{1} z_{1}^{2}}{x_{1} x_{1}^{*}}-\beta w_{1} z_{1} z_{2}-\alpha_{1} w_{1} z_{1} z_{3}+\beta w_{2} z_{1} z_{2}-\alpha_{2} w_{2} z_{2} z_{3}+\alpha_{1} w_{3} z_{1} z_{3}+\alpha_{2} w_{3} z_{2} z_{3}$

Now, choosing $w_{1}=w_{2}=w_{3}=1$, and putting these values in equation (3.3), we get:

$$
\dot{V}_{1}=-\frac{\theta z_{1}^{2}}{x_{1} x_{1}^{*}} \leq 0
$$

It is clear, by LaSalle's invariance principle, that $\left\{E_{4}\right\}$ is the only invariant set in $B \subset R_{+}^{3}$. Thus, by LaSalle-Lyapunov theorem $E_{4}$ is globally asymptotically stable. This completes the proof of theorem.

Remark 3.3. Equation (3.4) represents that $\dot{V}_{1}(t)=0$ on the set where $x_{1}=$ $x_{1}^{*}$. Since, $\dot{x}_{1}(t) \neq 0$ on $x_{1}=x_{1}^{*}$ unless $x_{2}=x_{2}^{*}$ and $y=y^{*}$, thus the largest positive invariant set is equilibrium point $\left(x_{1}^{*}, x_{2}^{*}, y^{*}\right)$. So that the LyapunovLaSalle theorem (Hale, 1980) implies that $\left(x_{1}^{*}, x_{2}^{*}, y^{*}\right)$ is globally asymptotically stable in $R_{+}^{3}$.

Now, we come to our original model, i.e., Model 2; where we will discuss the boundedness and the dynamical behaviour of the Model 2 . 
3.2. Analysis of Model 2: Now, we will show that all the solutions of the Model 2 are bounded. The boundedness of the solutions can be achieved by the following lemma.

Lemma 3.4. All the solutions of the Model 2 will lie in the following region as $t \longrightarrow \infty$ :

$$
\begin{gathered}
B_{2}=\left\{\left(x_{1}, x_{2}, y, C, U\right) \in R_{+}^{5}: 0 \leq x_{1}+x_{2}+y \leq M_{1}, m_{3} \leq C \leq M_{2}, m_{4} \leq U \leq\right. \\
\left.M_{3}, x_{1} \geq m_{1}, x_{1}+x_{2} \geq m_{2}\right\}
\end{gathered}
$$

where $M_{i}(i=1,2,3)$ and $m_{j}(j=1,2,3,4)$ are given in proof of the lemma.

Proof. Let us consider the following function:

$$
W(t)=x_{1}(t)+x_{2}(t)+y(t)
$$

then from equations (2.4)-(2.6), we get:

$$
\dot{W}(t) \leq \theta-d_{1} x_{1}-d x_{2}-d_{3} y
$$

if $\theta_{1}=\min \left(d_{1}, d, d_{3}\right)$ then by usual comparison theorem, we get following expression as $t \longrightarrow \infty$ :

$$
W \leq \frac{\theta}{\theta_{1}}=M_{1}(\text { say })
$$

hence

$$
x_{1}(t)+x_{2}(t)+y(t) \leq \frac{\theta}{\theta_{1}}
$$

From equation (2.7) and equation(2.8) we obtain:

$$
\frac{d C}{d t} \leq Q-\alpha C
$$

and

$$
\frac{d U}{d t} \leq \delta M_{1} M_{2}-m U
$$

then by usual comparison theorem,we get the following expressions as $t \longrightarrow \infty$ :

$$
C(t) \leq \frac{Q}{\alpha}=M_{2}(\text { say })
$$

and

$$
U(t) \leq \frac{\delta M_{1} M_{2}}{m}=M_{3}(\text { say })
$$

Again from equation(2.1),we get

$$
\frac{d x_{1}}{d t} \geq \theta-\eta_{1} x_{1}
$$

where

then we have

$$
\eta_{1}=\frac{\beta \theta}{\theta_{1}}+\frac{\alpha_{1} \theta}{\theta_{1}}+\frac{r_{1} Q}{\theta_{2}}+d_{1}
$$

hence

$$
\liminf _{t \longrightarrow \infty} x_{1}(t) \geq \frac{\theta}{\eta_{1}}
$$

$$
x_{1 \min } \geq \frac{\theta}{\eta_{1}}=m_{1}(\text { say })
$$


Similarly, we can calculated the minimum values for $x_{1}+x_{2}, C, U$. The calculated minimum values of $x_{1}+x_{2}, C$ and $U$ are given as:

$$
\begin{gathered}
x_{1 \text { min }}+x_{2 m i n} \geq \frac{\theta}{3 \eta_{2}}=m_{2}(\text { say }) \\
C_{\text {min }} \geq \frac{Q \theta_{1}}{2 \delta \theta+\alpha \theta_{1}}=m_{3}(\text { say }) \\
U_{\text {min }} \geq \frac{C_{\text {min }} \delta \theta}{3 m \eta_{2}}=m_{4}(\text { say })
\end{gathered}
$$

where,

$$
\eta_{2}=\max \left\{d_{1}, d, \alpha_{1} y_{\max }, \alpha_{2} y_{\max }, r_{1} U_{\max }, r_{2} U_{\max }\right\}
$$

This completes the proof of the lemma.

Now, we will discuss the feasibility and local stability of boundary equilibrium points and global stability of interior equilibrium point of the Model 2. We have considered the following set of equilibrium points: $E_{0}(0,0,0, C, 0), E_{1}\left(\bar{x}_{1}, \bar{x}_{2}, \bar{y}, 0,0\right)$, $E^{*}\left(x_{1}^{*}, x_{2}^{*}, y^{*}, C^{*}, U^{*}\right)$ and local behavior of equilibrium points $E_{0}$ and $E_{1}$ is given as follows:

- $E_{0}\left(0,0,0, \frac{Q}{\alpha}, 0\right)$ is always feasible. Corresponding to this equilibrium point we have the eigen values $-d_{1},-\alpha,-m, 0,0$.Thus $E_{0}$ is unstable.

- $E_{1}\left(\bar{x}_{1}, \bar{x}_{2}, \bar{y}, 0,0\right)$, the feasibility of this point is obvious. The characteristic equation corresponding to the equilibrium point $E_{1}$ is given as:

$$
\lambda^{5}+A \lambda^{4}+B \lambda^{3}+C \lambda^{2}+D \lambda+E=0
$$

where,

$$
\begin{aligned}
& A=\beta x_{2}+\alpha_{1} y+d_{1}+\alpha+m+\delta\left(x_{1}+x_{2}\right) \\
& B=\left(\beta x_{2}+\alpha_{1} y+d_{1}\right)\left(\alpha+m+\delta\left(x_{1}+x_{2}\right)\right)+m\left(\alpha+\delta\left(x_{1}+x_{2}\right)\right)+\alpha_{2}^{2} x_{2} y+ \\
& \beta^{2} x_{1} x_{2}+\alpha_{1}^{2} x_{1} y \\
& C=\left(\beta x_{2}+\alpha_{1} y+d_{1}\right)\left(\alpha m+\delta m\left(x_{1}+x_{2}\right)+\alpha_{2}^{2} x_{2} y\right)+\left(\alpha_{2}^{2} x_{2} y+\beta^{2} x_{1} x_{2}+\right. \\
& \left.\alpha_{1}^{2} x_{1} y\right)\left(\alpha+m+\delta\left(x_{1}+x_{2}\right)\right) \\
& D=\alpha_{2}^{2} x_{2} y\left(\beta x_{2}+\alpha_{1} y+d_{1}\right)\left(\alpha+m+\delta\left(x_{1}+x_{2}\right)\right)+m\left(\alpha_{2}^{2} x_{2} y+\beta^{2} x_{1} x_{2}+\right. \\
& \left.\alpha_{1}^{2} x_{1} y\right)\left(\alpha+\delta\left(x_{1}+x_{2}\right)\right) \\
& E=\alpha_{2}^{2} x_{2} y m\left(\beta x_{2}+\alpha_{1} y+d_{1}\right)\left(\alpha+\delta\left(x_{1}+x_{2}\right)\right) .
\end{aligned}
$$

For the convenience, we have dropped the bars from $x_{1}, x_{2}$ and $y$. It is clear that all the coefficients of characteristic equation are positive. Thus, by RouthHurwitz criteria $E_{1}$ is locally asymptotically stable if the following conditions hold:

$$
A>0,\left|\begin{array}{cc}
A & C \\
1 & B
\end{array}\right|>0,\left|\begin{array}{ccc}
A & C & E \\
1 & B & D \\
0 & A & C
\end{array}\right|>0
$$

Now, we will show the existence of the interior equilibrium point $E^{*}$ for the Model 2. The non-trivial positive equilibrium point can be found by the following system of equations:

$$
\begin{gathered}
\theta-\beta x_{1}^{*} x_{2}^{*}-\alpha_{1} x_{1}^{*} y^{*}-r_{1} U^{*} x_{1}^{*}-d_{1} x_{1}^{*}=0 \\
\beta x_{1}^{*}-\alpha_{2} x_{2}^{*} y^{*}-r_{2} U^{*}-d=0 \\
\alpha_{1} x_{1}^{*}+\alpha_{2} x_{2}^{*}-d_{3}=0
\end{gathered}
$$




$$
\begin{gathered}
Q-C^{*}\left(\alpha-\delta\left(x_{1}^{*}+x_{2}^{*}\right)\right)=0 \\
\delta C^{*}\left(x_{1}^{*}+x_{2}^{*}\right)-m U^{*}=0
\end{gathered}
$$

Thus on solving the above set of equations we get the positive interior equilibrium point $E^{*}=\left(x_{1}^{*}, x_{2}^{*}, y^{*}, C^{*}, U^{*}\right)$, where :

$$
\begin{gathered}
x_{1}^{*}=\frac{d_{3}}{\alpha_{1}}-\frac{\alpha_{2} x_{2}^{*}}{\alpha_{1}} \\
C^{*}=\frac{Q}{\alpha+\delta\left(x_{1}^{*}+x_{2}^{*}\right)} \\
y^{*}=\frac{\beta x_{1}^{*}}{\alpha_{2}}-\frac{r_{2} U^{*}}{\alpha_{2}}-\frac{d}{\alpha_{2}},
\end{gathered}
$$

and on solving the following isoclines, we can find $x_{2}^{*}$ and $U^{*}$ :

$$
\begin{gathered}
F\left(x_{2}, U\right)=k_{1} x_{2}+k_{2} U+k_{3} U x_{2}-k_{4}=0 \\
G\left(x_{2}, U\right)=l_{1} U+l_{2} x_{2} U+l_{3} x_{2}-l_{4}=0
\end{gathered}
$$

where, $k_{1}=\frac{\beta d_{3}}{\alpha_{1}}-d+\frac{d_{1} \alpha_{2}}{\alpha_{1}}, k_{2}=\frac{r_{2} d_{3}}{\alpha_{2}}-\frac{r_{1} d_{3}}{\alpha_{1}}-r_{2}, k_{3}=\frac{r_{1} \alpha_{2}}{\alpha_{1}}, k_{4}=-\frac{\beta d_{3}^{2}}{\alpha_{1} \alpha_{2}}+2 \frac{d d_{3}}{\alpha_{2}}-$ $\frac{d_{1} d_{3}}{\alpha_{1}}+\theta, l_{1}=m \alpha+\frac{\delta d_{3} m}{\alpha_{1}}, l_{2}=m \delta-\frac{m \delta \alpha_{2}}{\alpha_{1}}, l_{3}=\delta Q\left(\frac{\alpha_{2}}{\alpha_{1}}-1\right)$ and $l_{4}=\frac{d_{3} \delta Q}{\alpha_{1}}$.

From the equations (3.5) and (3.6) we obtain :

(a) $F\left(x_{2}, 0\right)=0$ i.e. $x_{2}^{*}=k_{4} / k_{1}=S_{11}$ (say)

(b) $F(0, U)=0$ i.e. $U^{*}=k_{4} / k_{2}=S_{12}$ (say),

and

2(a) $G\left(x_{2}, 0\right)=0$ i.e. $x_{2}^{*}=l_{4} / l_{3}=S_{21}$ (say)

2(b) $G(0, U)=0$ i.e. $U^{*}=l_{4} / l_{1}=S_{22}$ (say)

Thus two isoclines intersects in positive phase space if either of the following case holds:

(a) $S_{11}>S_{21}$ and $S_{12}<S_{22}$

(b) $S_{11}<S_{21}$ and $S_{12}>S_{22}$

The intersection of these two isoclines in positive phase space ensures the existence of $\left(x_{2}^{*}, U^{*}\right)$ and this point is unique if $U^{\prime}\left(x_{2}\right)<0$; hence, $E^{*}$ exists in positive phase plane [see, Figure 1].

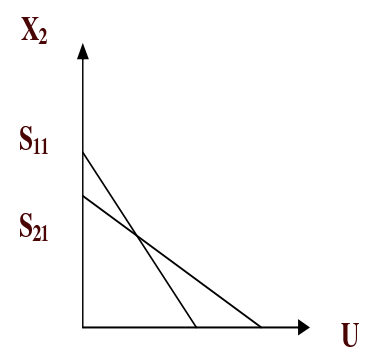

$\mathrm{S}_{12} \quad \mathrm{~S}_{22}$

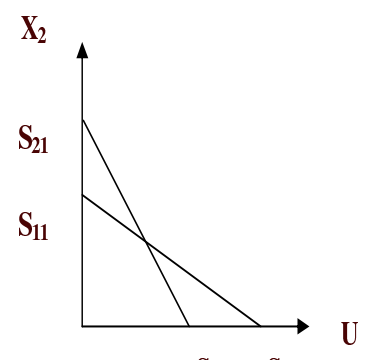

$\mathrm{S}_{22} \quad \mathrm{~S}_{12}$

Figure 1

Now, we will discuss the global stability of the interior equilibrium point $E^{*}$ by Lyapunov's direct method. This result has been established in the following theorem. 
Theorem 3.5. $E^{*}$ is globally asymptotically stable if $\beta<\beta^{*}, \alpha_{1}<\alpha_{1}^{*}, \alpha_{2}<\alpha_{2}^{*}$, $\delta<\delta^{*}, r_{1}<r_{1}^{*}, r_{2}<r_{2}^{*}, Q<Q^{*}$, and $m>m^{*}$, where $\beta^{*}, \alpha_{1}^{*}, \alpha_{2}^{*}, \delta^{*}, r_{1}^{*}, r_{2}^{*}, Q^{*}$ and $m^{*}$ are given in proof.

Proof. Let we consider the following positive definite function:

$$
\begin{aligned}
& V_{2}\left(x_{1}, x_{2}, y, C, U\right)=\frac{1}{2}\left(x_{1}-x_{1}^{*}\right)^{2}+ \frac{1}{2}\left(x_{2}-x_{2}^{*}\right)^{2}+\frac{1}{2}\left(y-y^{*}\right)^{2}+ \\
& \frac{1}{2}\left(C-C^{*}\right)^{2}+\frac{1}{2}\left(U-U^{*}\right)^{2}
\end{aligned}
$$

then the time derivative of above equation is given as:

$$
\dot{V}_{2}(t)=z_{1} \dot{x_{1}}+z_{2} \dot{x_{2}}+z_{3} \dot{y}+z_{4} \dot{C}+z_{5} \dot{U}
$$

where, $z_{1}=\left(x_{1}-x_{1}^{*}\right), z_{2}=\left(x_{2}-x_{2}^{*}\right), z_{3}=\left(y-y^{*}\right), z_{4}=\left(C-C^{*}\right)$, and $z_{5}=\left(U-U^{*}\right)$

Now, from equations (2.4)-(3.1) and from equation (3.8) we get:

$$
\dot{V}_{2}(t)=\dot{V}_{21}+\dot{V}_{22}+\dot{V}_{23}+\dot{V}_{24}+\dot{V}_{25}
$$

where

$$
\begin{aligned}
& \dot{V}_{21}=-\left\{\left(\beta x_{2}+\alpha_{1} y+r_{1} U+d_{1}\right) z_{1}^{2}+\beta x_{1}^{*} z_{1} z_{2}+\alpha_{1} x_{1}^{*} z_{1} z_{3}+r_{1} x_{1}^{*} z_{1} z_{5}\right\} \\
& \dot{V}_{22}=-\left\{\left(d+\alpha_{2} y+r_{2} U-\beta x_{1}\right) z_{2}^{2}-\beta x_{2}^{*} z_{1} z_{2}+\alpha_{2} x_{2}^{*} z_{2} z_{3}+r_{2} x_{2}^{*} z_{2} z_{5}\right\} \\
& \dot{V}_{23}=-\left\{\left(d_{3}-\alpha_{1} x_{1}-\alpha_{2} x_{2}\right) z_{3}^{2}-\alpha_{1} y^{*} z_{1} z_{3}-\alpha_{2} y^{*} z_{2} z_{3}\right\} \\
& \dot{V}_{24}=-\left\{\left(\alpha+\delta\left(x_{1}+x_{2}\right)\right) z_{4}^{2}+\delta C^{*} z_{1} z_{4}+\delta C^{*} z_{2} z_{4}\right\} \\
& \dot{V}_{25}=-\left\{m z_{5}^{2}-\delta\left(x_{1}+x_{2}\right) z_{4} z_{5}-\delta C^{*} z_{1} z_{5}-\delta C^{*} z_{2} z_{5}\right\} \\
& \text { thus we can write equation (3.9) in the following form: }
\end{aligned}
$$

$$
\begin{aligned}
\dot{V}_{2}=-\left\{a_{11} z_{1}^{2}+\right. & a_{12} z_{1} z_{2}+a_{13} z_{1} z_{3}+a_{15} z_{1} z_{5}+a_{22} z_{2}^{2}+a_{23} z_{2} z_{3}+a_{25} z_{2} z_{5} \\
& \left.+a_{33} z_{3}^{2}+a_{44} z_{4}^{2}+a_{14} z_{1} z_{4}+a_{24} z_{2} z_{4}+a_{55} z_{5}^{2}+a_{45} z_{4} z_{5}\right\}
\end{aligned}
$$

where,

$a_{11}=\left(\beta x_{2}+\alpha_{1} y+r_{1} U+d_{1}\right), a_{12}=\beta\left(x_{1}^{*}-x_{2}^{*}\right), a_{13}=\alpha_{1}\left(x_{1}^{*}-y^{*}\right), a_{24}=\delta C^{*}$,
$a_{15}=\left(r_{1} x_{1}^{*}-\delta C^{*}\right), a_{22}=\left(d+\alpha_{2} y+r_{2} U-\beta x_{1}\right), a_{23}=\alpha_{2}\left(x_{2}^{*}-y^{*}\right), a_{24}=\delta C^{*}$,
$a_{25}=\left(r_{2} x_{2}^{*}-\delta C^{*}\right), a_{33}=\left(d_{3}-\alpha_{1} x_{1}-\alpha_{2} x_{2}\right), a_{44}=\left(\alpha+\delta\left(x_{1}+x_{2}\right)\right), a_{45}=$
$-\delta\left(x_{1}+x_{2}\right), a_{55}=m$.
$\quad$ Now, we see that by Sylvester's criteria under the following conditions $\dot{V}_{2}(t)$ is negative definite.

(i) $4 a_{12}^{2}<a_{11} a_{22}$, (ii) $2 a_{13}^{2}<a_{11} a_{33}$, (iii) $3 a_{14}^{2}<a_{11} a_{44}$ (iv) $3 a_{15}^{2}<a_{11} a_{55}$, (v) $2 a_{23}^{2}<a_{22} a_{33}$, (vi) $3 a_{24}^{2}<a_{22} a_{44}$, (vii) $3 a_{25}^{2}<a_{22} a_{55}$, (viii) $9 a_{45}^{2}<4 a_{44} a_{55}$

Clearly, by Lyapunov's direct method $E^{*}$ is globally asymptotically stable.

Further, the above conditions (i)-(viii), obtained using Sylvester's criteria, can be rewritten respectively in the following form:

$$
\begin{gathered}
4 \beta^{2}\left(x_{1}^{*}-x_{2}^{*}\right)^{2}<\left(\beta x_{2 \max }+\alpha_{1} y_{\max }+r_{1} U_{\max }+d_{1}\right)\left(d+\alpha_{2} y_{\max }+r_{2} U_{\max }-\beta x_{1 \min }\right) \\
2 \alpha_{1}^{2}\left(x_{1}^{*}-y^{*}\right)^{2}<\left(\beta x_{2 \max }+r_{1} U_{\max }+\alpha_{1} y_{\max }+d_{1}\right)\left(d_{3}-\alpha_{1} x_{1 \min }-\alpha_{2} x_{2 \min }\right) \\
3 \delta^{2} C^{* 2}<(\beta .12) \\
3\left(x_{2 \max }+r_{1} U_{\max }+\alpha_{1} y_{\max }+d_{1}\right)\left(\alpha+\delta\left(x_{1 \max }+x_{2 \max }\right)\right)
\end{gathered}
$$




$$
\begin{aligned}
2 \alpha^{2}\left(x_{2}^{*}-y^{*}\right)^{2}< & \left(d+r_{2} U_{\max }-\beta x_{1 \min }+\alpha_{2} y_{\max }\right)\left(d_{3}-\alpha_{1} x_{1 \min }-\alpha_{2} x_{2 \min }\right) \\
3 \delta^{2} C^{* 2}< & \left(d+\alpha_{2} y_{\max }+r_{2} U_{\max }-\beta x_{1 \min }\right)\left(\alpha+\delta\left(x_{1 \max }+x_{2 \max }\right)\right) \\
& 3\left(r_{2} x_{2}^{*}-\delta C^{*}\right)^{2}<m\left(d+\alpha_{2} y_{\max }+r_{2} U_{\max }-\beta x_{1 \min }\right) \\
& 9 \delta^{2}\left(x_{1 \min }+x_{2 \min }\right)^{2}<4 m \alpha+4 m \delta\left(x_{1 \max }+x_{2 \max }\right)
\end{aligned}
$$

where $x_{1 \max }, x_{\max }, y_{\max }, C_{\max }, U_{\max }, x_{1 \min }, x_{2 \min }, C_{\min }$ can be obtained from region of attraction in lemma 3.2. Here, we have taken above inequalities in such a way that left hand side becomes minimum and the right hand side becomes maximum. This particular case represents the weakest situation for the inequalities to be held and ,moreover, if inequalities are dissatisfied in this situation then the paths starting in $B_{2}$ will not go to interior equilibrium point. Thus, under this particular situation we will break the above inequalities (3.11)-(3.18) to obtain the threshold conditions depending upon toxicant, disease and predation related parameters.

Now, on rearranging inequality (3.11), we obtain:

$$
f_{1}(\beta)=\pi_{11} \beta^{2}+\pi_{12} \beta-\pi_{13}<0
$$

where,

$$
\begin{aligned}
& \pi_{11}=4\left(x_{1}^{*}-x_{2}^{*}\right)^{2}+x_{1 \min } x_{2 \max }, \\
& \pi_{12}=x_{1 \min }\left(\alpha_{1} y_{\max }+r_{1} U_{\max }+d_{1}\right)-x_{2 \max }\left(d+\alpha_{2} y_{\max }+r_{2} U_{\max }\right) \\
& \pi_{13}=\left(d+\alpha_{2} y_{\max }+r_{2} U_{\max }\right)\left(\alpha_{1} y_{\max }+r_{1} U_{\max }+d_{1}\right)
\end{aligned}
$$

by theory of equations there exists a positive root of $f_{1}(\beta)=0$, say it is $\beta=\beta^{*}$. Then, clearly inequality (3.19) holds when $\beta<\beta^{*}$.

Now, on rearranging inequality (3.12), we obtain the following result:

$$
f_{2}\left(\alpha_{1}\right)=\pi_{21} \alpha_{1}^{2}+\pi_{22} \alpha_{1}-\pi_{23}<0
$$

where,

$$
\begin{aligned}
& \pi_{21}=2\left(x_{1}^{*}-y^{*}\right)^{2}+x_{1 \min } y_{\max } \\
& \pi_{22}=x_{1 \min }\left(\beta x_{2 \max }+r_{1} U_{\max }+d_{1}\right)-y_{\max }\left(d_{3}-\alpha_{2} x_{2 \min }\right) \\
& \pi_{23}=\left(\beta x_{2 \max }+r_{1} U_{\max }+d_{1}\right)\left(d_{3}-\alpha_{2} x_{2 \min }\right)
\end{aligned}
$$

by theory of equations there exists a positive root of $f_{2}\left(\alpha_{1}\right)=0$, say it is $\alpha_{1}=\alpha_{1}^{*}$. Then, clearly inequality (3.20) holds when $\alpha_{1}<\alpha_{1}^{*}$.

Now, on rearranging inequality (3.13), we obtain:

$$
f_{3}(\delta)=\pi_{31} \delta^{2}-\pi_{32} \delta-\pi_{33}<0
$$

where,

$$
\begin{aligned}
& \pi_{31}=3 C^{* 2} \\
& \pi_{23}=\left(\beta x_{2 \max }+\alpha_{1} y_{\max }+r_{1} U_{\max }+d_{1}\right)\left(x_{1 \max }+x_{2 \max }\right) \\
& \pi_{33}=\alpha\left(\beta x_{2 \max }+\alpha_{1} y_{\max }+r_{1} U_{\max }+d_{1}\right)
\end{aligned}
$$

by theory of equations there exists a positive root of $f_{3}(\delta)=0$, say it is $\delta=\delta^{*}$. Then, clearly inequality (3.21) holds when $\delta<\delta^{*}$.

Now, on rearranging inequality (3.14), we obtain that inequality (3.14) holds when $r_{1}<r_{1}^{*}$, where

$$
r_{1}^{*}=\frac{1}{x_{1}^{*}}\left\{\left(m\left(\beta x_{2 \max }+\alpha_{1} y_{\max }+r_{1} U_{\max }+d_{1}\right) / 3\right)^{1 / 2}+\delta C^{*}\right\}
$$


Now, on rearranging inequality (3.15), we obtain the following result:

$$
f_{4}\left(\alpha_{2}\right)=\pi_{41} \alpha_{2}^{2}+\pi_{42} \alpha_{2}-\pi_{43}<0
$$

where,

$\pi_{41}=2\left(x_{2}^{*}-y^{*}\right)^{2}+y_{\max } x_{2 \min }$

$\pi_{42}=x_{2 \min }\left(d+r_{2} U_{\max }-\beta x_{1 \min }\right)-y_{\max }\left(d_{3}-\alpha_{1} x_{1 \min }\right)$

$\pi_{43}=\left(d+r_{2} U_{\max }-\beta x_{1 \min }\right)\left(d_{3}-\alpha_{1} x_{1 \min }\right)$

by theory of equations there exists a positive root of $f_{4}\left(\alpha_{2}\right)=0$, say it is $\alpha_{2}=\alpha_{2}^{*}$.

Then, clearly inequality (3.22) holds when $\alpha_{2}<\alpha_{2}^{*}$.

Now, on rearranging inequality (3.16), we obtain that inequality (3.16) holds when $Q<Q^{*}$, where

$Q^{*}=\left(\alpha / \delta+x_{1}^{*}+x_{2}^{*}\right)\left\{\left(\left(d+\alpha_{2} y_{\max }+r_{2} U_{\max }-\beta x_{1 \min }\right)\left(\alpha+\delta\left(x_{1 \max }+x_{2 \max }\right)\right) / 3\right)\right\}^{1 / 2}$

Now, on rearranging inequality (3.17), we obtain that inequality (3.17) holds when $r_{2}<r_{2}^{*}$, where

$$
r_{2}^{*}=\frac{1}{x_{2}^{*}}\left\{\left(m\left(d+\alpha_{2} y_{\max }+r_{1} U_{\max }-\beta x_{1 \min }\right) / 3\right)^{1 / 2}+\delta C^{*}\right\}
$$

Now, on rearranging inequality (3.18), we obtain that inequality (3.18) holds when $m>m^{*}$, where

$$
m^{*}=\frac{9 \delta^{2}\left(x_{1 \min }+x_{2 \min }\right)^{2}}{4 \alpha+4 \delta\left(x_{1 \max }+x_{2 \max }\right)}
$$

Its clear that $E^{*}$ is globally asymptotically stable if $\beta<\beta^{*}, \alpha_{1}<\alpha_{1}^{*}, \alpha_{2}<\alpha_{2}^{*}$, $\delta<\delta^{*}, r_{1}<r_{1}^{*}, r_{2}<r_{2}^{*}, Q<Q^{*}$, and $m>m^{*}$.

This completes the proof of the theorem.

Remark 3.6. Biologically, we can say that if the disease contact rate, predation rates among susceptible and infected prey populations, toxicant uptake rate by prey population, rates at which susceptible and infected prey populations are decreasing due to toxicant and exogenous input rate of toxicant in environment are under upper threshold values; and natural wash-out rate of the toxicant from the organism is higher than the lower threshold value then interior equilibria of the system given by model 2 is globally asymptotically stable. The threshold values that we have obtained in the above theorem are important to ensure the global stability of the interior equilibrium point. If any one of the threshold conditions is disturbed then paths starting in interior of the region $B_{2}$ can approach to the boundaries.

\section{Numerical ExAmple}

We consider the following set of parameters:

$\theta=50, \alpha_{1}=0.01, \alpha_{2}=0.02, \beta=0.01, r_{1}=0.001, r_{2}=0.01, d_{1}=0.01, d=0.1$, $d_{3}=1, Q=10, \alpha=0.2, \delta=0.1, m=0.2$

Then we get the following results:

(1) Region of attraction for the Model 1 is:

$$
B_{1}=\left\{\left(x_{1}, x_{2}, y\right) \in R_{+}^{3}: 0 \leq x_{1}+x_{2}+y \leq 500\right\}
$$


and the interior equilibrium point of Model 1 is $E_{4}=(9.9010,45.0495,44.5050)$ [see Figure 3].

(2) Region of attraction for the Model 2 is:

$$
B_{2}=\left\{\left(x_{1}, x_{2}, y, C, U\right) \in R_{+}^{5}: 0 \leq x_{1}+x_{2}+y \leq 500,0 \leq C+U \leq 50\right\}
$$

and corresponding interior equilibrium point is $E^{*}=(10.2994,44.8528,22.3470$, $1.7499,48.2509)$. From theorem $3.2, E^{*}$ is globally asymptotically stable if $\beta<\beta^{*}$, $\alpha_{1}<\alpha_{1}^{*}, \alpha_{2}<\alpha_{2}^{*}, \delta<\delta^{*}, r_{1}<r_{1}^{*}, r_{2}<r_{2}^{*}, Q<Q^{*}$, and $m>m^{*}$, where

$$
\begin{gathered}
\beta^{*}=1.0229, \alpha_{1}^{*}=1.0229, \delta^{*}=1.0 e+003 * 6.0034, r_{1}^{*}=0.2032, \alpha_{2}^{*}=0.3554, \\
Q^{*}=1.0708 e+003, r_{2}^{*}=0.0226, m^{*}=0.000623
\end{gathered}
$$

Hence, $E^{*}$ is globally asymptotically stable [see Figure 2]. All the feasible local and global stability conditions have also been verified by these numerical values. For this example, both the systems are long term persistent under the basic results given by Theorem (3.1) and Theorem (3.2).

Remark 4.1. By the analysis of both the Model 1 and the Model 2, it is clear that due to adverse effect of toxicant the equilibria level of prey-predator system, when prey is infected due to some disease, decreases.

Remark 4.2. Numerically, it has been observed that both the susceptible and infected prey population can not increase simultaneously; on the account of this fact, we can say that if susceptible prey population increases then correspondingly infected prey population will decrease and vice-versa [see Figure 2 and Figure 3].

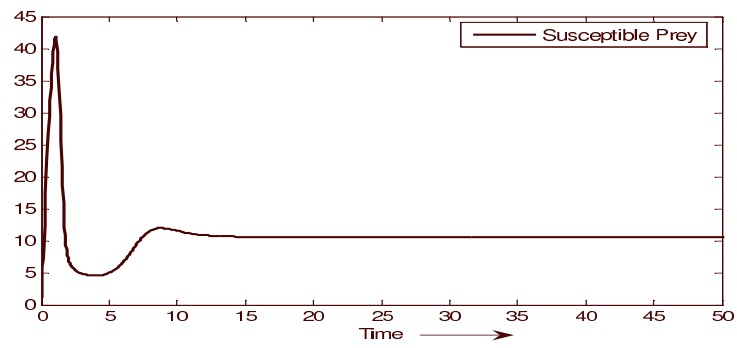

Figure 2(a)

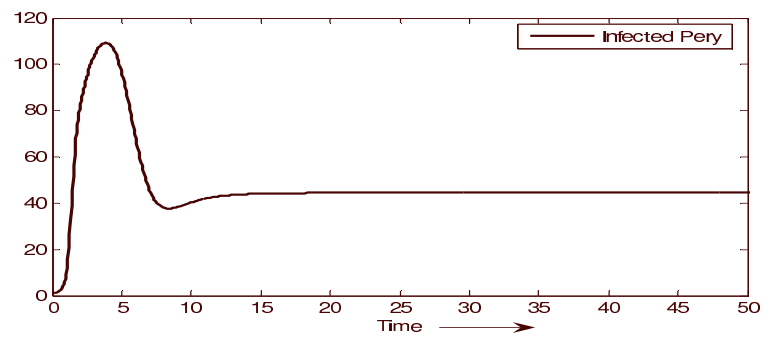

Figure 2(b)

\section{Conclusion}

We have proposed a mathematical model to study the effect of toxicant in preypredator system when the prey population is already subjected to some disease. 


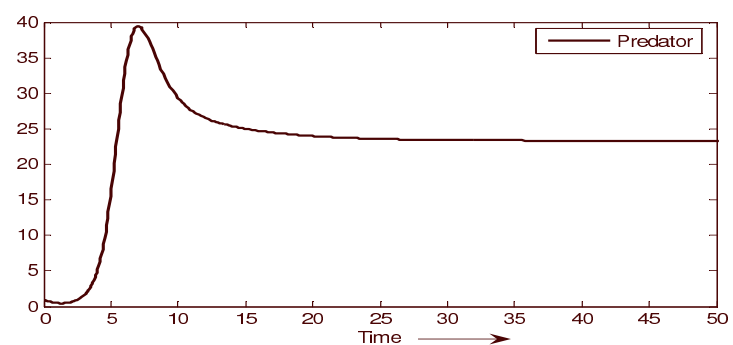

Figure 2(c)

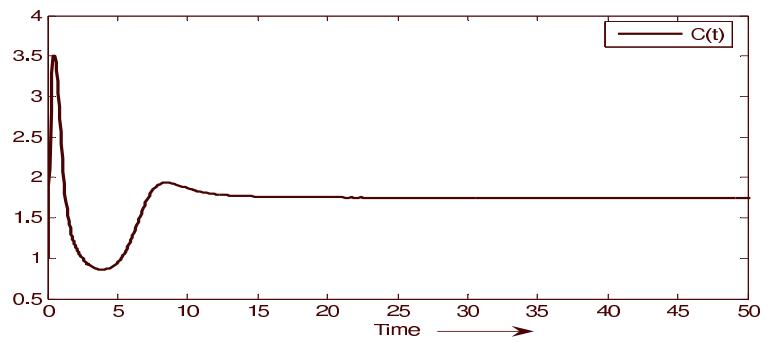

Figure 2(d)

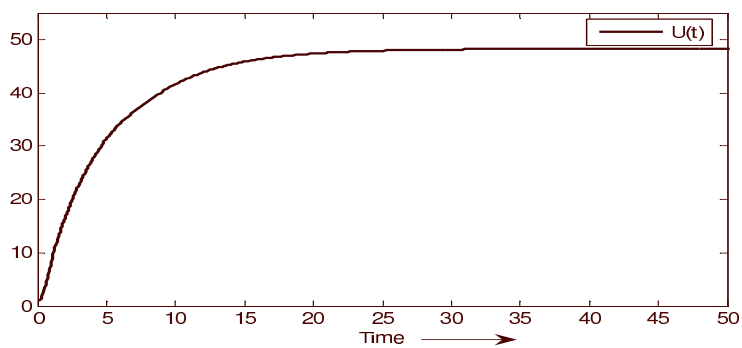

Figure 2(e)

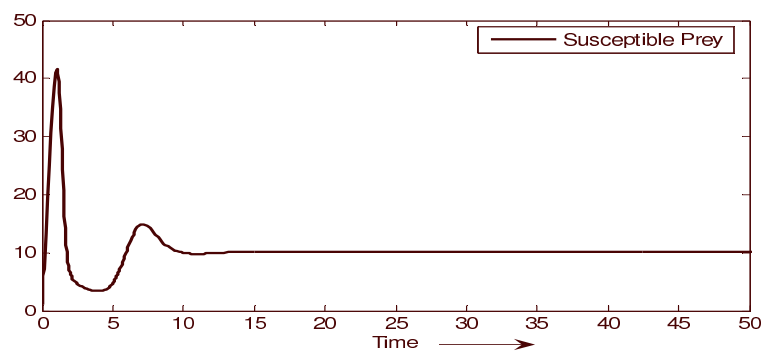

Figure 3(a)

It has been determined that when the effect of toxicant is not considered in the prey species, Model 1 is globally asymptotically stable, for any positive non-zero recruitment rate in susceptible population. Further, the interior equilibrium point of the Model 1 is the only invariant set in solution space in positive phase plane. Again, when we consider the effect of toxicant in prey population then it has been observed that the interior equilibrium point is the only invariant set in solution 

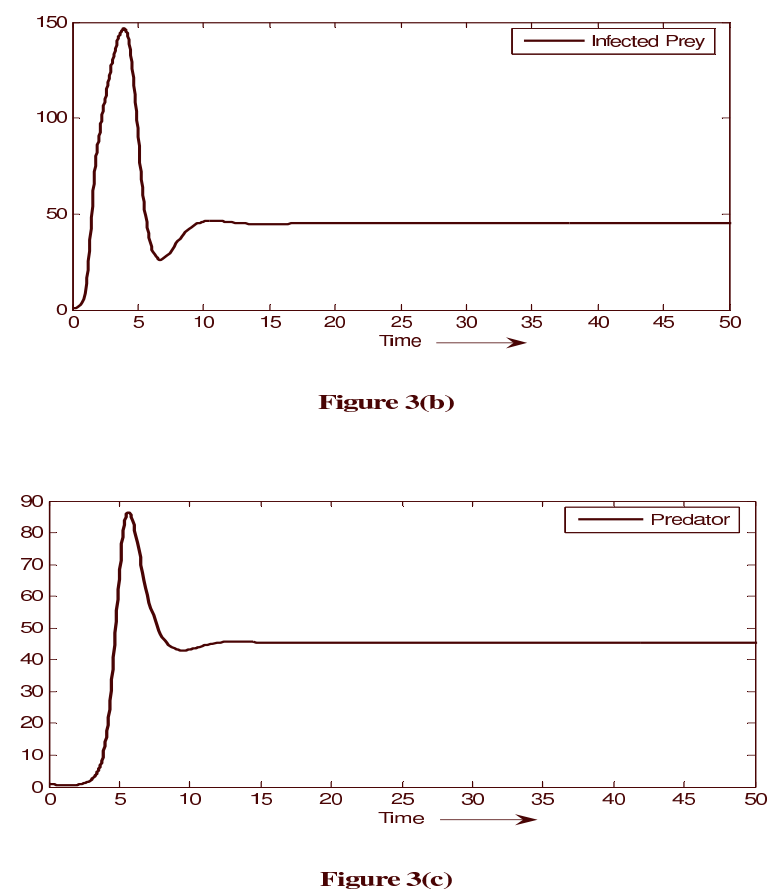

space, but, global stability of the system is now depending upon system parameters. Moreover, it has been observed that when the disease contact rate (i.e., $\beta)$, predation rates among susceptible and infected prey populations (i.e., $\alpha_{1}$ and $\alpha_{2}$ ), toxicant uptake rate by prey population (i.e., $\delta$ ), rates at which susceptible and infected prey populations are decreasing due to toxicant (i.e., $r_{1}$ and $r_{2}$ ) and exogenous input rate (i.e, Q) of toxicant in environment are under upper threshold values; and natural wash-out rate of the toxicant from the organism (i.e, $\mathrm{m}$ ) is higher than the lower threshold value then prey and predator population can survive for long period. Finally, numerical verifications of analytic results have shown in figures 1-3.

\section{REFERENCES}

1. R.M. Anderson, R.M. May, Epidemiology and genetics in the Coevolution of parasites and hosts, Proc.R.Soc.Lond., B 219 (1983), 281. 1]

2. R.M. Anderson, R.M. May, The invasion, persistence and spread of infections disease within animal and plant communities, Philos. Trans. R. Soc. Lond., B 314 (1986), 533. 1

3. R.M. Anderson, R.M. May, The population dynamics of the microparasites and their invertebrate hosts, Philos. Trans. R. Soc. Lond., B 291 (1981), 451. 1

4. R.A. Adler, R.C. Brunet, The dynamics of the simultaneous infections with altered susceptibilities, Theoret. Popul. Biol., 40, 369, (1991). 1

5. M. Begon, R.G. Bowers, Beyond host pathogen dynamics, in : B.T. Grenfell, A.P. Dooson (Editors), Ecology of Infectious Diseases in Natural Population, Cambridge University, Cambridge, P. 478, 1995. 1 
6. M. Begon, R.G. Bowers, Host-host pathogen models and microbial pest control : the effect of host self-regulation, J. Theoret. Biol. 169 (1995), 275. 1

7. S. Busenberg, P. Van den, P.Driessche, Analysis of a disease transmission model in a population with varying size, J. Math Biol. 28 (1990), 257. 1

8. Joydip Dhar, A Prey-Predator model with diffusion and a supplementry resource for the prey in a two patch environment, J.math. modl. and Analy. 9 (2004), 9-24. 1

9. B. Dubey, Modelling the interaction of two biological species in a polluted environment, J. Math. Anal. Appl. 246 (2000), 58-79.

10. B. Dubey, Modelling the depletion and conservation of resources : effect of two interacting populations, Ecol. Model. 101 (1997), 123-136. 1

11. B. Dubey, J. Hussain, Non-linear models for the survival of two competing species in a polluted environment, Non-lin. Anal. Rl. Worl. Appl. 4 (2003), 21-44. 1

12. H.I. Freedman, J.B. Shukla, Models for the effect of toxicant in single species and predatorprey system, J. Math. Biol. 30 (1991), 15-30. 1

13. L.Q. Gao, H.W. Hethcote, Disease transmission models with density-dependent demographics, J. Math. Biol. 30 (1992), 717. 1

14. A.G. Gordon, E. Gorham, Ecological aspects of air pollution from iron sintering plant of Wawa, Can. J.Bot. 41 (1963), 1063-1078. 1

15. J.K. Hale, Ordinary differential equations, 2nd Ed., Kriegor, Basel, 1980.

16. R.D. Holt, J. Pickering, Infectious disease and species co-existence : A model of LoktaVoltera form, Am. Nat. 126 (1986), 196. 1

17. K.P. Hadeler, H.I. Freeman, Predator-Prey Population with parasitic infection, J. Math. Biol. 27 (1989), 609. 1

18. H.W.Hethcote, Three basic epidemiological models, in : S.A. Levin, T.G. Hallom, L.J. Gross (Eds.) Applied Mathematical Ecology, Biomath., Vol. 18 (1989), 119-144. 1

19. S.A. Levin, Some approaches to the modelling of co-evolutionary interactions, in : $M$. Nitecki (Ed.), Coevolution, University of Chicago, Chicago, 1983. 1

20. S.A. Levin, Coevolution, in : H.I. Freedman, C. Strobeck (Eds), Population Biology, lect. not. in Biomath., Vol. 52 , Springer-Heidelberg, 1983. 1

21. B. Liu, L. Chen, Y. Zhang, The effect of impulsive toxicant input on a population in a polluted environment, Journ. of biol.Sys. 11(3) (2003), 265-274. 1

22. D.Mukherjee, Persistence and global stability of a population in a polluted environment with delay, J. Biol. Sys. 10 (2002), 225-232. 1

23. J. Mena-Lorca, H.W. Hethcote, Dynamic models of infections diseases as regulator of population sizes, J. Math. Biol. 30, (1992), 693. 1

24. M.N. Rao, H.V.N. Rao, Air pollution, Tata McGraw Hill publishing Co. Ltd., New Del, 1989. 1

25. J.B. Shukla, B. Dubey, Simultaneous effect of two toxicants on biological species : A mathematical model, J. Biol. System 4 (1996), 109-130. 1

26. J.B. Shukla, B. Dubey, Modelling the depletion and conservation of forestry resources : Effect of population and pollution, J. Math. Biol. 36 (1997), 71-94. 1

27. S.C. Santra, A.C. Samual, Air quality of Kalyani township (Nadia, West Bengal) and its impact on surrounding vegetation, Ind. J. Env. Healt. 44(1) (2000) 71-76. 1

${ }^{1}$ School of Mathematics and Allied Sciences, Jiwaji University Gwalior (M.P.)474011, INDIA.

E-mail address: sudeepasinha@yahoo.com

2 School of Mathematics and Allied Sciences, Jiwaji University Gwalior (M.P.)474011, INDIA.

E-mail address: misra_op58@yahoo.co.in 
3 Department of Applied Sciences, ABV-Indian Institute of Information TechNOLOGY AND MANAGEMENT, GWALIOR(M.P.)-474011, INDIA.

E-mail address: jdhar@iitm.ac.in 